\title{
Assets, Aims, Actions, Alignment, Audit: 5As in Diabetes
}

\author{
Ameya Joshi ${ }^{1}$ \\ ${ }^{1}$ Department of Endocrinology, Bhaktivedanta Hospital, Mumbai, India
}

J Soc Health Diab 2019;7:89-90

\begin{abstract}
Address for correspondence Ameya Joshi, Bhaktivedanta Hospital, Bhaktivedanta Swami Marg, Siddhi Vinayak Nagar, Sector 1, Srishti Complex, Mira Road, Mira Bhayandar, Maharashtra 401107, India (e-mail: ameyaable@gmail.com).
\end{abstract}
Abstract
Keywords
- diabetes
- informed decision-making
- logical empiricism
- patient-centered care
- pragmatic logic

This brief communication shares the importance of the Alignment of the 3As, that is Assets, Aims, and Actions, while managing diabetes. It describes the essence of asset analysis, target decision-making, and therapeutic planning strategies. Calling for regular Audits of diabetes care, it reinforces the importance of this policy at the level of the person with diabetes, diabetes care provider, and healthcare system.

\section{Patient-centered Care}

The concept of patient-centered care has become integral to the practice of diabetes care. While various definitions of patient-centered care have been offered, the most popular is "care that is respectful of and responsive to individual patient preferences, needs, and values" and that "ensures that patient values guide all clinical decisions."1 The patient-centered model of care is seen in ancient tests as well as modern literature. Related frameworks such as informed decision-making, shared decision-making, and responsible patient-centered care have all been used to enhance the quality of diabetes care.,3 In spite of this, however, persons afflicted with diabetes, and their family members, complain of inadequate understanding and empathy from healthcare providers. Similar frustration is voiced by diabetes care providers, who feel that persons with diabetes do not take adequate responsibility of their health.

We propose a simple framework to help align patient and physician expectations, needs and activities, into a concordant relationship, so as to minimize friction and achieve optimal outcomes. We term this the $5 \mathrm{~A}$ alignment rubric.

\section{Assets}

The first A stands for Asset assessment. This includes an analysis of the strengths, limitations, and vulnerabilities of the person with diabetes, the diabetes care team, and the healthcare system. An accurate asset mapping will allow an understanding of the needs and expectations of the person afflicted with diabetes, and help anticipate the efforts and energy required to fulfil them. The diabetes care team can then decide how best to leverage resources of the healthcare system, the family, and society at large, to maximize efficiency and output.

\section{Aims}

This brings us to the second A, which stands for Aims. Assets' assessment also contributes to decisions regarding Aims (targets), which are framed according to biomedical health status as well as psychosocial capabilities. Persons with high levels of motivation, good diabetes literacy and numeracy, as well as strong social support (optimal Assets) may aim for tight glycemic control, while those with suboptimal Assets may prefer lax levels of metabolic control.

Aims and targets also depend upon Assets of the healthcare system. Diabetes care settings which have adequate personnel and facilities for glucose monitoring may aim for tight glucose control, while those who are challenged in terms of human or physical infrastructure may aim for relaxed targets.

\section{Actions}

Once assets have been assessed, and aims decided, one may plan the strategies necessary to achieve those aims using available assets. Thus, the third A, or actions, follows and builds upon the first two, that is, Assets and Aims. Actions 
must be appropriate for the Assets that are available, and must be geared toward achieving predecided Aims. This implies that diagnostic, screening, or therapeutic strategies which may be relevant for a particular clinical situation, or healthcare setting, may not be ideal for another.

\section{Alignment}

The key to achieving optimal outcomes is a fourth A or Alignment. Alignment of Assets, Aims, and Actions is necessary if one is to achieve effective and efficient attainment of health. As all the three As are dynamic, so is their alignment. The 4th $\mathrm{A}$, therefore, not only aims to check this at regular intervals but also orders mid-course correction if needed.

\section{Audit}

Auditing of one's assets, aims, and actions should be undertaken by persons with diabetes as well as their diabetes care providers. Biomedical as well as psychosocial parameters using laboratory reported and self- reported measures can be monitored to quantify the person's or provider's progress along the path to health. An example of misalignment, uncovered by audit, would be a therapeutic strategy designed on the sliding scale, aiming to achieve "ideal" glucose targets in an indoor care setting, without ensuring availability of trained nursing staff, glucose monitoring devices, or facilities to manage hypoglycemia.

\section{Conclusion}

The three As, Assets, Aims, and Actions, if managed in Alignment with each other, under the watchful gaze of Audit, should help achieve optimal therapeutic outcomes in persons with diabetes, compassion satisfaction among their caregivers, and professional satisfaction among diabetes care providers.

\section{Conflict of Interest}

None declared.

\section{References}

1 Institute of Medicine (US) Committee on quality of health care in America. Crossing The Quality Chasm: A New Health System For The 21st Century. Washington DC: The National Academies Press; 2001

2 Kalra S, Baruah MP, Kalra B. Diabetes care: evolution of philosophy. Indian J Endocrinol Metab 2017;21(4):495-497

3 KalraS,BaruahMP,UnnikrishnanAG.Responsiblepatient-centered care. Indian J Endocrinol Metab 2017;21(3):365-366 